PROCEEDINGS OF THE

AMERICAN MATHEMATICAL SOCIETY

Volume 127, Number 5, Pages 1293-1300

S 0002-9939(99)04817-0

Article electronically published on January 27, 1999

\title{
ON COVERING MULTIPLICITY
}

\author{
ZHI-WEI SUN
}

(Communicated by David E. Rohrlich)

\begin{abstract}
Let $A=\left\{a_{s}+n_{s} \mathbb{Z}\right\}_{s=1}^{k}$ be a system of arithmetic sequences which forms an $m$-cover of $\mathbb{Z}$ (i.e. every integer belongs at least to $m$ members of $A$ ). In this paper we show the following surprising properties of $A$ : (a) For each $J \subseteq\{1, \cdots, k\}$ there exist at least $m$ subsets $I$ of $\{1, \cdots, k\}$ with $I \neq J$ such that $\sum_{s \in I} 1 / n_{s}-\sum_{s \in J} 1 / n_{s} \in \mathbb{Z}$. (b) If $A$ forms a minimal $m$-cover of $\mathbb{Z}$, then for any $t=1, \cdots, k$ there is an $\alpha_{t} \in[0,1)$ such that for every $r=0,1, \cdots, n_{t}-1$ there exists an $I \subseteq\{1, \cdots, k\} \backslash\{t\}$ for which $\left[\sum_{s \in I} 1 / n_{s}\right] \geqslant m-1$ and $\left\{\sum_{s \in I} 1 / n_{s}\right\}=\left(\alpha_{t}+r\right) / n_{t}$.
\end{abstract}

\section{INTRODUCTION}

For integer $a$ and positive integer $n$ we call

$$
a(n)=\{x \in \mathbb{Z}: x \equiv a(\bmod n)\}=a+n \mathbb{Z}
$$

an arithmetic sequence with common difference $n$ or a residue class with modulus $n$. For a finite system

$$
A=\left\{a_{s}\left(n_{s}\right)\right\}_{s=1}^{k}
$$

of such sets, we define its covering multiplicity by

$$
m(A)=\inf _{x \in \mathbb{Z}}|S(x)|
$$

where $S(x)=\left\{1 \leqslant s \leqslant k: x \equiv a_{s}\left(\bmod n_{s}\right)\right\}$. It is easy to show that

$$
\sum_{s=1}^{k} \frac{1}{n_{s}} \geqslant m(A),
$$

and the equality holds if and only if (1) covers each integer exactly $m$ times for some $m=1,2,3, \cdots$. (Cf. [S2], [S4].)

Let $m$ be a nonnegative integer. If system (1) has covering multiplicity at least $m$, then we call (1) an $m$-cover (of $\mathbb{Z}$ ). A minimal $m$-cover (of $\mathbb{Z}$ ) is an $m$-cover whose proper subsystems are not. If $|S(x)|=m$ for all $x \in \mathbb{Z}$, then we say that $A$ forms an exact $m$-cover (of $\mathbb{Z}$ ). Notice that an exact 1 -cover is a partition of $\mathbb{Z}$ into (finitely many) periodic sets. The Chinese Remainder Theorem tells that the intersection of residue classes $a_{1}\left(n_{1}\right), \cdots, a_{k}\left(n_{k}\right)$ is empty if and only if two of them are disjoint. So, as a dual question, when (1) forms a 1-cover is fundamental and

Received by the editors August 13, 1997.

1991 Mathematics Subject Classification. Primary 11B25; Secondary 11A07, 11B75, 11 D68.

Supported by the National Natural Science Foundation of the People's Republic of China and the Return-from-abroad Foundation of the Chinese Educational Committee.

(C)1999 American Mathematical Society 
important. In fact, 1-covers and exact $m$-covers (especially exact 1-covers) have been investigated for many years; also some famous conjectures remain open. (See R. K. Guy $[\mathrm{G}]$.)

Now we introduce some notation. As usual, if $m$ and $n$ are integers, then $(m, n)$ represents the greatest common divisor of $m$ and $n$. For a real number $x$, we set $\left(\begin{array}{l}x \\ 0\end{array}\right)=1$ and let $\left(\begin{array}{l}x \\ n\end{array}\right)=\prod_{j=0}^{n-1} \frac{x-j}{n-j}$ for $n=1,2,3, \cdots ;$ also $[x]$ and $\{x\}$ denote the integral and the fractional parts of $x$ respectively.

In this paper we study the covering multiplicity of a general system of residue classes. Our main result is as follows.

Theorem 1. Let (1) be a system of arithmetic sequences, and let $J$ be a subset of $\{1, \cdots, k\}$. Put $J^{-}=\{1, \cdots, k\} \backslash J$.

(i) For any $m_{1}, \cdots, m_{k} \in \mathbb{Z}$ we have

$$
\left|\left\{I \subseteq\{1, \cdots, k\}: I \neq J \&\left\{\sum_{s \in I} \frac{m_{s}}{n_{s}}\right\}=\left\{\sum_{s \in J} \frac{m_{s}}{n_{s}}\right\}\right\}\right| \geqslant m(A) .
$$

(ii) Suppose $\emptyset \neq J \subseteq S(x)$ for some $x \in \mathbb{Z}$ with $|S(x)|=m(A)$. For each $s \in J^{-}$ let $m_{s}$ be a positive integer prime to $n_{s}$. Then there exists an $\alpha \in[0,1)$ such that

$$
\begin{aligned}
& \left\{\left\{\sum_{s \in I} \frac{m_{s}}{n_{s}}\right\}: I \subseteq J^{-},\left[\sum_{s \in I} \frac{m_{s}}{n_{s}}\right] \geqslant m(A)-|J|\right\} \\
& \supseteq\left\{\frac{a}{N(J)}: 0 \leqslant a<N(J),\{a\}=\alpha\right\},
\end{aligned}
$$

where $N(J)$ denotes the least common multiple of those $n_{s}$ with $s \in J$.

In view of Theorem 1 , an $m$-cover $A=\left\{a_{s}\left(n_{s}\right)\right\}_{s=1}^{k}$ possesses the following properties:

(a) For each $J \subseteq\{1, \cdots, k\}$, there exist at least $m$ subsets $I$ of $\{1, \cdots, k\}$ with $I \neq J$ such that $\sum_{s \in I} 1 / n_{s}-\sum_{s \in J} 1 / n_{s} \in \mathbb{Z}$.

(b) If $A$ forms a minimal $m$-cover of $\mathbb{Z}$, then for any $t=1, \cdots, k$ there is an $\alpha_{t} \in[0,1)$ such that, for every $r=0,1, \cdots, n_{t}-1$, there exists an $I \subseteq\{1, \cdots, k\} \backslash\{t\}$ for which $\left[\sum_{s \in I} 1 / n_{s}\right] \geqslant m-1$ and $\left\{\sum_{s \in I} 1 / n_{s}\right\}=\left(\alpha_{t}+r\right) / n_{t}$.

Part (i) of Theorem 1 can be strengthened in the case $J=\emptyset$. By Theorem 1 , if (1) forms a 1-cover, then $\sum_{s \in I} 1 / n_{s} \in \mathbb{Z}$ for some nonempty subset $I$ of $\{1, \cdots, k\}$, which is the main result of M. Z. Zhang [Z] obtained by means of the Riemann zeta function. For an exact $m$-cover (1), the author proved in [S1] that for each $n=0,1, \cdots, m$ there exist at least $\left(\begin{array}{c}m \\ n\end{array}\right)$ subsets $I$ of $\{1, \cdots, k\}$ with $\sum_{s \in I} 1 / n_{s}=n$. When (1) is an $m$-cover and $m_{1}, \cdots, m_{k}$ are positive integers, it was shown in [S3] that there are at least $m$ positive integers in the form $\sum_{s \in I} m_{s} / n_{s}$ where $I \subseteq\{1, \cdots, k\}$; we even conjecture that there exist nonempty subsets $I_{1}, \cdots, I_{m}$ of $\{1, \cdots, k\}$ for which $I_{1} \subset \cdots \subset I_{m}$ and $\sum_{s \in I_{t}} m_{s} / n_{s} \in \mathbb{Z}$ for all $t=1, \cdots, m$.

The first part of Theorem 1 yields

Corollary 1. Let (1) be an $m$-cover of $\mathbb{Z}$ and $m_{1}, \cdots, m_{k}$ any integers. Then

$$
\left|\left\{\left\{\sum_{s \in I} \frac{m_{s}}{n_{s}}\right\}: I \subseteq\{1, \cdots, k\}\right\}\right| \leqslant \frac{2^{k}}{m+1} .
$$

Proof. By part (i) of Theorem 1 , for any $J \subseteq\{1, \cdots, k\}$ there are at least $m+1$ subsets $I$ of $\{1, \cdots, k\}$ with $\left\{\sum_{s \in I} m_{s} / n_{s}\right\}=\left\{\sum_{s \in J} m_{s} / n_{s}\right\}$. Since $\{1, \cdots, k\}$ has exactly $2^{k}$ subsets, Corollary 1 follows immediately. 
Remark 1. A conjecture of P. Erdös proved by R. B. Crittenden and C. L. Vanden Eynden $[\mathrm{CV}]$ states that (1) forms a 1 -cover of $\mathbb{Z}$ if it covers $1, \cdots, 2^{k}$. In [S2], [S3] the author showed that (1) forms an $m$-cover of $\mathbb{Z}$ if there exist $W$ consecutive integers each of which lies in at least $m$ members of (1), where $W$ is the least integer equal to the left hand side of (6) for some integers $m_{1}, \cdots, m_{k}$ prime to $n_{1}, \cdots, n_{k}$ respectively.

As for part (ii) of Theorem 1 we should mention the following result obtained by the author ([S4]) recently: Let (1) be an exact $m$-cover of $\mathbb{Z}$, and $J$ a nonempty subset of $\{1, \cdots, k\}$ with $\left(n_{s}, n_{t}\right) \mid a_{s}-a_{t}$ for all $s, t \in J$ (i.e. $\emptyset \neq J \subseteq S(x)$ for some $x \in \mathbb{Z}$ ). Then

$$
\left|\left\{I \subseteq J^{-}:\left\{\sum_{s \in I} \frac{1}{n_{s}}\right\}=\frac{a}{N(J)}\right\}\right| \geqslant \frac{\prod_{s \in J} n_{s}}{N(J)}
$$

for every $a=0,1, \cdots, N(J)-1$, and

$$
\left|\left\{I \subseteq J^{-}: \sum_{s \in I} \frac{1}{n_{s}}=\frac{a}{N(J)}\right\}\right| \geqslant\left(\begin{array}{c}
m-1 \\
{[a / N(J)]}
\end{array}\right)
$$

for all $a=0,1,2, \cdots$ if $|J|=1$.

Corollary 2. Let (1) be an $m$-cover of $\mathbb{Z}$ with $n_{1} \leqslant \cdots \leqslant n_{k-1} \leqslant n_{k}$. Suppose that $B=\left\{a_{s}\left(n_{s}\right)\right\}_{s=1}^{k-1}$ fails to be an $m$-cover of $\mathbb{Z}$. If $\sum_{s=1}^{k-1} 1 / n_{s}=m$, then $n_{k-1}=n_{k}>1$ and

$$
\left\{\sum_{s \in I} \frac{1}{n_{s}}: I \subseteq\{1, \cdots, k-1\}\right\} \supseteq\left\{\frac{r}{n_{k}}: r=0,1, \cdots, n_{k}-1\right\} .
$$

Proof. Assume that $\sum_{s=1}^{k-1} 1 / n_{s}=m$. By part (ii) of Theorem 1 there exists an $\alpha \in[0,1)$ such that

$$
\begin{gathered}
\left\{\left\{\sum_{s \in I} \frac{1}{n_{s}}\right\}: I \subseteq\{1, \cdots, k-1\},\left[\sum_{s \in I} \frac{1}{n_{s}}\right] \geqslant m-1\right\} \\
\supseteq\left\{\frac{a}{n_{k}}: 0 \leqslant a<n_{k},\{a\}=\alpha\right\} .
\end{gathered}
$$

Thus

$$
\begin{aligned}
& \left\{\sum_{s \in J} \frac{1}{n_{s}}: J \subseteq\{1, \cdots, k-1\}, \sum_{s \in J} \frac{1}{n_{s}} \notin \mathbb{Z}\right\} \\
\supseteq & \left\{\sum_{s=1}^{k-1} \frac{1}{n_{s}}-\sum_{s \in I} \frac{1}{n_{s}}: I \subseteq\{1, \cdots, k-1\}, m-1<\sum_{s \in I} \frac{1}{n_{s}}<m=\sum_{s=1}^{k-1} \frac{1}{n_{s}}\right\} \\
= & \left\{1-\left\{\sum_{s \in I} \frac{1}{n_{s}}\right\}: I \subseteq\{1, \cdots, k-1\},\left[\sum_{s \in I} \frac{1}{n_{s}}\right] \geqslant m-1\right\} \backslash\{1\} \\
\supseteq & \left\{1-\frac{a}{n_{k}}: 0 \leqslant a<n_{k},\{a\}=\alpha\right\} \backslash\{1\}=\left\{\frac{b}{n_{k}}: 0<b<n_{k},\{b\}=\{-\alpha\}\right\} .
\end{aligned}
$$

Observe that (7) follows if $\alpha=0$. Since $B$ doesn't form an $m$-cover of $\mathbb{Z}$, we cannot have $n_{1}=\cdots=n_{k-1}=1$ (otherwise $k-1=\sum_{s=1}^{k-1} 1 / n_{s}=m$ ). So $n_{k} \geqslant n_{k-1}>1$; 
hence by the above for some nonempty $J \subseteq\{1, \cdots, k-1\}$ we have

$$
\frac{1}{n_{k-1}} \leqslant \min _{s \in J} \frac{1}{n_{s}} \leqslant \sum_{s \in J} \frac{1}{n_{s}}=\frac{1-\alpha}{n_{k}} \leqslant \frac{1}{n_{k}} \leqslant \frac{1}{n_{k-1}} .
$$

Therefore $n_{k}=n_{k-1}$ and $\alpha=0$. We are done.

Remark 2. Let (1) be an $m$-cover of $\mathbb{Z}$ with $n_{1} \leqslant \cdots \leqslant n_{k-1}<n_{k}$. By part (iv) of Theorem I of [S3], $\sum_{s=1}^{k-1} 1 / n_{s} \geqslant m$. In view of Corollary 2, if $\left\{a_{s}\left(n_{s}\right)\right\}_{s=1}^{k-1}$ fails to be an $m$-cover of $\mathbb{Z}$, then $\sum_{s=1}^{k-1} 1 / n_{s}$ must be greater than $m$. This extends and improves a confirmed conjecture of Erdös which states that $\sum_{s=1}^{k} 1 / n_{s}>1$ for any 1 -cover (1) with $1<n_{1}<\cdots<n_{k-1}<n_{k}$ (see [E] and [G]).

Corollary 3. Let (1) be an $m$-cover of $\mathbb{Z}$, and $J$ a nonempty subset of $\{1, \cdots, k\}$ with $\left|\left\{s \in J^{-}: x \in a_{s}\left(n_{s}\right)\right\}\right|=m-|J|$ for some $x \in \mathbb{Z}$. Let $\varepsilon_{s} \in\{1,-1\}$ for those $s \in J^{-}$. Then

$$
\left|\left\{\left\{\sum_{s \in I} \frac{\varepsilon_{s}}{n_{s}}\right\}: I \subseteq J^{-}\right\}\right| \geqslant N(J) .
$$

Proof. This follows immediately from the second part of Theorem 1.

Remark 3. With the help of a local-global result proved in [S2], in 1994 the author found Corollary 3 in the case $|J|=1$ (see Section 3 of [S3]).

Corollary 4. Let (1) be a minimal $m$-cover of $\mathbb{Z}$, and $m_{1}, \cdots, m_{k}$ any positive integers prime to $n_{1}, \cdots, n_{k}$ respectively. Then for every $t=1, \cdots, k$ all the numbers $0,1 / n_{t}, \cdots,\left(n_{t}-1\right) / n_{t}$ lie in the set

$$
\left\{\left\{\sum_{s \in I} \frac{m_{s}}{n_{s}}-\sum_{s \in J} \frac{m_{s}}{n_{s}}\right\}: I, J \subseteq\{1, \cdots, k\} \backslash\{t\} \& \sum_{s \in I} \frac{m_{s}}{n_{s}}, \sum_{s \in J} \frac{m_{s}}{n_{s}} \geqslant m-1\right\} .
$$

Proof. By part (ii) of Theorem 1 there is an $\alpha_{t} \in[0,1)$ such that

$$
\left\{\left\{\sum_{s \in I} \frac{m_{s}}{n_{s}}\right\}: I \subseteq\{1, \cdots, k\} \backslash\{t\},\left[\sum_{s \in I} \frac{m_{s}}{n_{s}}\right] \geqslant m-1\right\}
$$

contains $S_{t}=\left\{a / n_{t}: 0 \leqslant a<n_{t},\{a\}=\alpha_{t}\right\}$. As $r / n_{t}=\left(\alpha_{t}+r\right) / n_{t}-\alpha_{t} / n_{t}$ for each $r=0,1, \cdots, n_{t}-1$, the desired result follows.

Remark 4. In [S3] the author was able to prove Corollary 4 with $\sum_{s \in J} m_{s} / n_{s} \geqslant$ $m-1$ in (9) replaced by $\sum_{s \in J} m_{s} / n_{s} \geqslant m-2$.

\section{Proof of Theorem 1}

Let's recall a key result given by the author in [S2].

Proposition 1. Let $\mathcal{A}=\left\{\alpha_{s}+\beta_{s} \mathbb{Z}\right\}_{s=1}^{k}$ where $\alpha_{1}, \cdots, \alpha_{k}$ are real numbers and $\beta_{1}, \cdots, \beta_{k}$ are positive reals. Let $m$ be a positive integer. Then $\mathcal{A}$ forms an $m$-cover of $\mathbb{Z}$ (i.e. $\left|\left\{1 \leqslant s \leqslant k:\left(x-\alpha_{s}\right) / \beta_{s} \in \mathbb{Z}\right\}\right| \geqslant m$ for all $\left.x \in \mathbb{Z}\right)$ if and only if

$$
\sum_{\substack{I \subseteq\{1, \cdots, k\} \\
\left\{\sum_{s \in I}^{\left.1 / \beta_{s}\right\}=\theta}\right.}}(-1)^{|I|}\left(\begin{array}{c}
{\left[\sum_{s \in I} 1 / \beta_{s}\right]} \\
n
\end{array}\right) e^{2 \pi i \sum_{s \in I} \alpha_{s} / \beta_{s}}=0
$$

holds for all $\theta \in[0,1)$ and $n=0,1, \cdots, m-1$. 
Lemma 1. Let $k, m, n$ be positive integers with $k>m-n \geqslant 0$. Then (1) forms an $m$-cover of $\mathbb{Z}$ if and only if for each $I \subseteq\{1, \cdots, k\}$ with $|I|=m-n$ system $A_{I}=\left\{a_{s}\left(n_{s}\right)\right\}_{s \in I^{-}}$forms an $n$-cover of $\mathbb{Z}$.

Proof. If (1) is an $m$-cover of $\mathbb{Z}$ and $I$ is a subset of $\{1, \cdots, k\}$ with $|I|=m-n$, then for any integer $x$ we have

$$
\left|\left\{s \in I^{-}: x \equiv a_{s}\left(\bmod n_{s}\right)\right\}\right| \geqslant m-|I|=n
$$

therefore $A_{I}$ is an $n$-cover of $\mathbb{Z}$.

Now suppose that $A_{I}$ forms an $n$-cover of $\mathbb{Z}$ for all $I \subseteq\{1, \cdots, k\}$ with $|I|=$ $m-n$. Let's show that $A=A_{\emptyset}$ forms an $m$-cover of $\mathbb{Z}$. Assume on the contrary that for some integer $x$ set $J=\left\{1 \leqslant s \leqslant k: x \equiv a_{s}\left(\bmod n_{s}\right)\right\}$ has cardinality $l<m$. Choose a subset $I$ of $\{1, \cdots, k\}$ with cardinality $m-n$ such that either $I \subseteq J$ or $I \supseteq J$. Observe that $x$ belongs to less than $n$ members of $A_{I}$. This contradiction ends our proof.

Remark 5. Apparently for (1) to be an $m$-cover of $\mathbb{Z}$ it is necessary that $k \geqslant m$.

Proof of part (i) of Theorem 1. It suffices to handle the case $m=m(A)>0$.

At first we assume that $n_{1}, \cdots, n_{k}$ are all greater than one. Since $m \leqslant \sum_{s=1}^{k} 1 / n_{s}$ $\leqslant k / 2$, either $J$ or $J^{-}$has cardinality not less than $m$.

Case 1. $\left|J^{-}\right| \geqslant m$. Among $I \subseteq\{1, \cdots, k\}$ with $\left\{\sum_{s \in I} m_{s} / n_{s}\right\}=\left\{\sum_{s \in J} m_{s} / n_{s}\right\}$, we select a $J_{0}$ with the least cardinality. Apparently $\left|J_{0}^{-}\right| \geqslant\left|J^{-}\right| \geqslant m$. Let $I_{0}=\left\{s_{1}, \cdots, s_{m-1}\right\}$ be a subset of $\{1, \cdots, k\}$ with $\left|I_{0}\right|=m-1$ and $I_{0} \cap J_{0}=\emptyset$. By Lemma 1 and Remark 5, system $\left\{a_{s}\left(n_{s}\right)\right\}_{s \in I_{0}^{-}}$forms a 1-cover of $\mathbb{Z}$ and hence so does $\mathcal{A}_{0}=\left\{a_{s}+\left(n_{s} / m_{s}\right) \mathbb{Z}\right\}_{s \in I_{0}^{-}}$. As $J_{0} \subseteq I_{0}^{-}$, by Proposition 1 or Theorem 2 of [S2] there is a $J_{1} \subseteq I_{0}^{-}$for which $J_{1} \neq J_{0}$ and $\left\{\sum_{s \in J_{1}} m_{s} / n_{s}\right\}=$ $\left\{\sum_{s \in J_{0}} m_{s} / n_{s}\right\}$. According to the choice of $J_{0}$ we must have $J_{1} \not \nexists J_{0}$. Choose $t_{1} \in J_{1} \backslash J_{0}$ and put $I_{1}=\left\{t_{1}, s_{2}, \cdots, s_{m-1}\right\}$. Observe that $I_{1} \cap J_{0}=\emptyset$. Since $\mathcal{A}_{1}=\left\{a_{s}+\left(n_{s} / m_{s}\right) \mathbb{Z}\right\}_{s \in I_{1}^{-}}$forms a 1 -cover of $\mathbb{Z}$, there exists a $J_{2} \subseteq I_{1}^{-}$with $J_{2} \neq J_{0}$ such that $\left\{\sum_{s \in J_{2}} m_{s} / n_{s}\right\}=\left\{\sum_{s \in J_{0}} m_{s} / n_{s}\right\}$. Choose $t_{2} \in J_{2} \backslash J_{0}$ and put $I_{2}=$ $\left\{t_{1}, t_{2}, s_{3}, \cdots, s_{m-1}\right\}$. Then continue this procedure to find $J_{3}, t_{3}, I_{3} ; \cdots ; J_{m-1}$, $t_{m-1}, I_{m-1} ; J_{m}, t_{m}$ in the same way. Apparently $J_{1}, J_{2}, \cdots, J_{m}$ are all different from $J_{0}$. If $1 \leqslant i<j \leqslant m$, then $t_{i} \in J_{i} \backslash J_{j}$ because $t_{i} \in I_{j-1}$ and $J_{j} \cap I_{j-1}=\emptyset$. So the $m+1$ subsets $J_{0}, J_{1}, J_{2}, \cdots, J_{m}$ of $\{1, \cdots, k\}$ are distinct; therefore

$$
\begin{aligned}
& \left|\left\{I \subseteq\{1, \cdots, k\}: I \neq J \& \sum_{s \in I} \frac{m_{s}}{n_{s}}-\sum_{s \in J} \frac{m_{s}}{n_{s}} \in \mathbb{Z}\right\}\right| \\
& \quad \geqslant\left|\left\{J_{i}: 0 \leqslant i \leqslant m \& J_{i} \neq J\right\}\right| \geqslant m .
\end{aligned}
$$

Case 2. $|J| \geqslant m$, i.e. $\left|\left(J^{-}\right)^{-}\right| \geqslant m$. It follows from the above that

$$
\left|\left\{I \subseteq\{1, \cdots, k\}: I \neq J^{-} \& \sum_{s \in I} \frac{m_{s}}{n_{s}}-\sum_{s \in J^{-}} \frac{m_{s}}{n_{s}} \in \mathbb{Z}\right\}\right| \geqslant m
$$


Thus

$$
\begin{aligned}
& \left|\left\{I^{\prime} \subseteq\{1, \cdots, k\}: I^{\prime} \neq J \& \sum_{s \in I^{\prime}} \frac{m_{s}}{n_{s}}-\sum_{s \in J} \frac{m_{s}}{n_{s}} \in \mathbb{Z}\right\}\right| \\
= & \left|\left\{I^{-}: I \subseteq\{1, \cdots, k\}, I^{-} \neq J \& \sum_{s \in I^{-}} \frac{m_{s}}{n_{s}}-\sum_{s \in J} \frac{m_{s}}{n_{s}} \in \mathbb{Z}\right\}\right| \\
= & \left|\left\{I \subseteq\{1, \cdots, k\}: I \neq J^{-} \& \sum_{s \in I} \frac{m_{s}}{n_{s}}-\sum_{s \in J^{-}} \frac{m_{s}}{n_{s}} \in \mathbb{Z}\right\}\right| \geqslant m .
\end{aligned}
$$

So far we have proven (4) in both cases.

Next let's consider the situation in which $K=\left\{1 \leqslant s \leqslant k: n_{s}=1\right\}$ is nonempty. If $|K|<m$, then $\left\{a_{s}\left(n_{s}\right)\right\}_{s \in K^{-}}$forms an $m-|K|$-cover of $\mathbb{Z}$ with all the moduli greater than one; hence by the above

$$
\left|\left\{I \subseteq K^{-}: \sum_{s \in I} \frac{m_{s}}{n_{s}}-\sum_{s \in J \backslash K} \frac{m_{s}}{n_{s}} \in \mathbb{Z}\right\}\right| \geqslant m-|K|+1 .
$$

Therefore

$$
\begin{aligned}
& \left|\left\{I \subseteq\{1, \cdots, k\}: \sum_{s \in I} \frac{m_{s}}{n_{s}}-\sum_{s \in J} \frac{m_{s}}{n_{s}} \in \mathbb{Z}\right\}\right| \\
= & \left|\left\{I \cup I^{\prime}: I \subseteq K, I^{\prime} \subseteq K^{-} \& \sum_{s \in I^{\prime}} \frac{m_{s}}{n_{s}}-\sum_{s \in J \backslash K} \frac{m_{s}}{n_{s}} \in \mathbb{Z}\right\}\right| \\
\geqslant & \left|\left\{I \cup I^{\prime}: I \subseteq K,|I| \leqslant 1, I^{\prime} \subseteq K^{-} \& \sum_{s \in I^{\prime}} \frac{m_{s}}{n_{s}}-\sum_{s \in J \backslash K} \frac{m_{s}}{n_{s}} \in \mathbb{Z}\right\}\right| \\
\geqslant & |K|+\left|\left\{I^{\prime} \subseteq K^{-}: \sum_{s \in I^{\prime}} \frac{m_{s}}{n_{s}}-\sum_{s \in J \backslash K} \frac{m_{s}}{n_{s}} \in \mathbb{Z}\right\}\right| \\
\geqslant & |K|+\max \{m-|K|+1,1\} \geqslant m+1 .
\end{aligned}
$$

This completes the proof.

Lemma 2. Let (1) be a system of arithmetic sequences, and $J$ a nonempty subset of $\{1, \cdots, k\}$ with $|J| \leqslant m(A)$ and $\bigcap_{s \in J} a_{s}\left(n_{s}\right) \neq \emptyset$. For each $s \in J^{-}$let $m_{s}$ be $a$ positive integer. Let $0 \leqslant a<N(J)$ and

$$
C(a)=\sum_{\substack{I \subseteq J^{-} \\
\left\{\sum_{s \in I} \frac{m_{s}}{n_{s}}\right\}=\frac{a}{N(J)}}}(-1)^{|I|}\left(\begin{array}{c}
{\left[\sum_{s \in I} \frac{m_{s}}{n_{s}}\right]} \\
m(A)-|J|
\end{array}\right) e^{2 \pi i \sum_{s \in I} \frac{m_{s}}{n_{s}}\left(a_{s}-a_{J}\right)}
$$

where $a_{J}$ is the unique integer in $\bigcap_{s \in J} a_{s}\left(n_{s}\right)$ with $0 \leqslant a_{J}<N(J)$. Then $C(a)=$ $C(\{a\})$.

Proof. Apparently it suffices to show $C(a)=C(a-1)$ providing $a \geqslant 1$. 
Let $m=m(A)$. Observe that the sequences $a_{s}+\left(n_{s} / m_{s}\right) \mathbb{Z}\left(s \in J^{-}\right)$together with $a_{J}+N(J) \mathbb{Z}$ form an $m-|J|+1$-cover of $\mathbb{Z}$. In view of Proposition 1 ,

$$
\begin{aligned}
& \sum_{I \subseteq J^{-}}(-1)^{|I|}\left(\begin{array}{c}
{\left[\sum_{s \in I} \frac{m_{s}}{n_{s}}\right]} \\
m-|J|
\end{array}\right) e^{2 \pi i \sum_{s \in I} \frac{a_{s} m_{s}}{n_{s}}} \\
& \left\{\sum_{s \in I} \frac{m_{s}}{n_{s}}\right\}=\frac{a}{N(J)} \\
& +\quad \sum_{I \subseteq J^{-}} \quad(-1)^{|I|+1}\left(\begin{array}{c}
{\left[\sum_{s \in I} \frac{m_{s}}{n_{s}}+\frac{1}{N(J)}\right]} \\
m-|J|
\end{array}\right) e^{2 \pi i\left(\sum_{s \in I} \frac{a_{s} m_{s}}{n_{s}}+\frac{a_{J}}{N(J)}\right)} \\
& \left\{\sum_{s \in I} \frac{m_{s}}{n_{s}}+\frac{1}{N(J)}\right\}=\frac{a}{N(J)}
\end{aligned}
$$

vanishes. So

$$
\begin{aligned}
& e^{2 \pi i a a_{J} / N(J)} C(a) \\
= & \sum_{\substack{I \subseteq J^{-} \\
\left\{\sum_{s \in I} \frac{m_{s}}{n_{s}}\right\}=\frac{a}{N(J)}}}(-1)^{|I|}\left(\begin{array}{c}
{\left[\sum_{s \in I} \frac{m_{s}}{n_{s}}\right]} \\
m-|J|
\end{array}\right) e^{2 \pi i \sum_{s \in I} \frac{a_{s} m_{s}}{n_{s}}} \\
= & \sum_{\substack{I \subseteq J^{-} \\
\left\{\sum_{s \in I} \frac{m_{s}}{n_{s}}\right\}=\frac{a-1}{N(J)}}}(-1)^{|I|}\left(\begin{array}{c}
\left.\left[\sum_{s \in I} \frac{m_{s}}{n_{s}}\right]+\frac{a}{N(J)}\right] \\
m-|J|
\end{array}\right) e^{2 \pi i\left(\sum_{s \in I} \frac{a_{s} m_{s}}{n_{s}}+\frac{a_{J}}{N(J)}\right)} \\
= & e^{2 \pi i a_{J} / N(J)} \sum_{\substack{I \subseteq J^{-} \\
\left\{\sum_{s \in I}\right.}}(-1)^{|I|}\left(\begin{array}{c}
{\left[\sum_{s \in I} n_{s} \frac{m_{s}}{n_{s}}\right]} \\
m-|J|
\end{array}\right) e^{2 \pi i \sum_{s \in I} \frac{a_{s} m_{s}}{n_{s}}} \\
= & e^{2 \pi i a_{J} / N(J)} e^{2 \pi i(a-1) a_{J} / N(J)} C(a-1)=e^{2 \pi i a a_{J} / N(J)} C(a-1) .
\end{aligned}
$$

Therefore $C(a)=C(a-1)$. We are done.

Remark 6. If we replace $m(A)-|J|$ in (11) by a smaller nonnegative integer $n$, then the new $C(a)$ will equal zero by Proposition 1 , because system $\left\{a_{s}+\left(n_{s} / m_{s}\right) \mathbb{Z}\right\}_{s \in J^{-}}$ forms an $m(A)-|J|$-cover of $\mathbb{Z}$.

Proof of part (ii) of Theorem 1. Since $\left|\left\{s \in J^{-}: x \in a_{s}\left(n_{s}\right)\right\}\right|=m(A)-|J|$ for some integer $x$ and $\left(m_{s}, n_{s}\right)=1$ for all $s \in J^{-}$, system $\left\{a_{s}+\left(n_{s} / m_{s}\right) \mathbb{Z}\right\}_{s \in J^{-}}$fails to form an $m(A)-|J|+1$-cover of $\mathbb{Z}$ as well as $\left\{a_{s}\left(n_{s}\right)\right\}_{s \in J^{-}}$. As $x \in \bigcap_{s \in J} a_{s}\left(n_{s}\right)$, there is a unique integer $a_{J}$ with $0 \leqslant a_{J}<N(J)$ such that $a_{J} \equiv a_{s}\left(\bmod n_{s}\right)$ for all $s \in J$. By Proposition 1 and Remark 6 there exists a $\theta \in[0,1)$ such that

$$
C(N(J) \theta) e^{2 \pi i a_{J} \theta}=\sum_{\substack{I \subseteq J^{-} \\
\left\{\sum_{s \in I} \frac{m_{s}}{n_{s}}\right\}=\theta}}(-1)^{|I|}\left(\begin{array}{c}
{\left[\sum_{s \in I} \frac{m_{s}}{n_{s}}\right]} \\
m(A)-|J|
\end{array}\right) e^{2 \pi i \sum_{s \in I} \frac{a_{s} m_{s}}{n_{s}}} \neq 0 .
$$

Put $\alpha=\{N(J) \theta\}$. If $0 \leqslant a<N(J)$ and $\{a\}=\alpha$, then $a-N(J) \theta \in \mathbb{Z}$ and hence $C(a)=C(N(J) \theta) \neq 0$ by Lemma 2; therefore $\left\{\sum_{s \in I} \frac{m_{s}}{n_{s}}\right\}=\frac{a}{N(J)}$ for some $I \subseteq J^{-}$ with $\left[\sum_{s \in I} \frac{m_{s}}{n_{s}}\right] \geqslant m(A)-|J|$. This concludes the proof.

\section{ACKNowledgement}

The author is indebted to the referee for his comments. 


\section{REFERENCES}

[CV] R. B. Crittenden and C. L. Vanden Eynden, Any $n$ arithmetic progressions covering the first $2^{n}$ integers cover all integers, Proc. Amer. Math. Soc. 24 (1970), 475-481. MR 41:3365

[E] P.Erdös, Problems and results in number theory, in: H. Halberstam and C. Holley, eds., Recent Progress in Analytic Number Theory, vol. 1, Academic Press, New York, 1981, pp. 1-13. MR 84j:10001

[G] R. K. Guy, Unsolved Problems in Number Theorey (2nd, ed.), Springer-Verlag, New York, 1994, pp. 251-256. MR 96e:11002

[S1] Z. W. Sun, On exactly $m$ times covers, Israel J. Math. 77 (1992), 345-348. MR 93k:11007

[S2] Z. W. Sun, Covering the integers by arithmetic sequences, Acta Arith. 72 (1995), 109-129. MR 96k:11013

[S3] Z. W. Sun, Covering the integers by arithmetic sequences II, Trans. Amer. Math. Soc. 348 (1996), 4279-4320. MR 97c:11011

[S4] Z. W. Sun, Exact $m$-covers and the linear form $\sum_{s=1}^{k} x_{s} / n_{s}$, Acta Arith. 81 (1997), 175198. CMP 97:14

[Z] M. Z. Zhang, A note on covering systems of residue classes, J. Sichuan Univ. (Nat. Sci. Ed.) 26 (1989), Special Issue, 185-188. MR 92c:11003

Department of Mathematics, Nanjing University, Nanjing 210093, People's Republic OF CHINA

E-mail address: zwsun@netra.nju.edu.cn 